Extension of previous rule-based explanation techniques has been required to generate critiques of this type.

\footnotetext{
* Support for this work was provided by the National Library of Medicine under Grant LM-03395, and by the Office of Naval Research under contract NR $049-479$. Computing facilities were provided by the SUMEXAIM resource under NIH Grant RR-00785. I also thank Ted Shortliffe and Bruce Buchanan for helpful comments on earlier drafts of this paper.

$\dagger$ Heuristic Programming Project, Departments of Medicine and Computer Science, Stanford University, Stanford, California 94305.
}

\title{
Computer Programs That Adjust Insulin Dosage for Diabetics Who Monitor Their Blood Glucose
}

Nat Pernick, M.D.*

This paper describes a set of computer programs that use Dr. Jay Skyler's algorithms to adjust insulin dosage for diabetic patients who monitor their blood glucose. These programs, which can easily be adapted to inexpensive microcomputers, choose the correct algorithms for pregnancy and six insulin dosing regimens, including the insulin pump. The programs also recommend blood glucoses to check; print patient messages about diet, illness, or calling a physician; graph glucose values by time of day; and calculate mean blood glucose, $M$ value, and the number of hypoglycemic episodes. These features, coupled with the ease of using the programs, may promote improved glycemic control in diabetics.

* The University of Michigan, Ann Arbor, Michigan 48109. 